\title{
Mineração
}

\section{Adsorção do corante têxtil amarelo remazol usando um dos rejeitos da fabricação da alumina como adsorvente}

\section{(Adsorption of remazol yellow dye using as adsorvent a solid waste from the production of alumina)}

\author{
Elizabeth Gonçalves Rutz \\ Mestranda do Programa de Pós-Graduação em Engenharia Ambiental - UFOP \\ E-mail: elizabethrutz@yahoo.com.br \\ Cornélio de Freitas Carvalho \\ Professor do Departamento de Química - UFOP.E-mail: cornelio@iceb.ufop.br \\ Carulini de Souza Pires \\ Graduanda do Curso de Farmácia - UFOP. E-mail: carulini@yahoo.com.br \\ Gustavo Lopes Curiel \\ Graduando do Curso de Engenharia Ambiental - UFOP.E-mail: gucuriel@hotmail.com
}

\section{Resumo}

Esse trabalho discute a possibilidade de utilização de um dos rejeitos da fabricação da alumina, pó retido no filtro eletrostático, na adsorção do corante amarelo remazol, usado rotineiramente na indústria têxtil. Os resultados mostram, para uma solução aquosa $50 \mathrm{mg} \mathrm{L}^{-1}$, cerca de 95 \% de remoção do corante em solução. O modelo de isoterma de Langmuir mostrou-se adequado para descrever o mecanismo de adsorção desse corante. A capacidade de adsorção do corante depende da sua estrutura e das interações corante-adsorvente.

Palavras-chave: Adsorção, alumina, amarelo remazol, Langmuir.

\begin{abstract}
This work discusses the possibility of using one of the residues retained in electrostatic filters during alumina production, in the adsorption of the remazol yellow dye which is commonly employed in the textile industry. The results presents a removal efficiency of $95 \%$ of the dye in an aqueous solution containing $50 \mathrm{mg} / \mathrm{L}$ of this substance. The Langmuir isotherm model showed to be adequate to describe the adsorption mechanism of this dye. The ability of the dye adsorption depends on its structure and the interaction dyeadsorbent.
\end{abstract}

Keywords: Adsorption, alumina, remazol yellow, Langmuir. 


\section{Introdução}

A indústria têxtil é uma das maiores produtoras de efluentes líquidos em virtude do grande consumo de água e produtos químicos, em especial nos processos de tingimento e acabamento (Leão et al., 1998). No processo de tingimento, são adicionados os corantes, empregados sob a forma de soluções ou dispersões aquosas, por indução com reativos apropriados ou por controle das condições físicas como temperatura e pH, conferindo cor aos tecidos (Andrade, 1999).

Além de causarem poluição visual e prejuízos à biota aquática, estudos recentes têm mostrado que algumas classes de corantes podem ser carcinogênicos e/ou mutagênicos (Kunz et alii, 2002).

Devido às implicações ambientais citadas, novas tecnologias têm sido estudadas para a degradação ou imobilização desses compostos em efluentes têxteis. Existe um crescente interesse no uso de materiais adsorventes sólidos de baixo custo para o tratamento de efluentes têxteis (Jain et alii, 2003), os quais oferecem uma série de vantagens dentro da sua simplicidade, rapidez e eficiência (Allen et alii, 2004).

Uma das etapas do processo industrial de extração de alumínio é a obtenção de alumina $\left(\mathrm{Al}_{2} \mathrm{O}_{3}\right)$. Nessa etapa, um dos rejeitos obtidos é um pó fino, proveniente de filtros eletrostáticos, que é descartado junto à lama vermelha em barragens e aterros industriais (Santos, 1997). O resíduo não satisfaz as especificações industriais para a sua utilização ou para a produção de alumínio. A quantidade produzida desse rejeito é de cerca de 1 a $2 \%$ da produção total de alumina (Gonçalves, 2003).

Esse trabalho busca estudar a possibilidade de uso de um dos rejeitos da fabricação da alumina, pó retido no filtro eletrostático, como adsorvente do corante amarelo remazol, usado rotineiramente na indústria têxtil.

\section{Materiais e métodos}

\section{Caracterização do rejeito da fabricação da alumina}

O rejeito da fabricação da alumina - pó retido no filtro eletrostático - foi caracterizado através de medidas de área superficial específica, análise granulométrica, basicidade, acidez e $\mathrm{pH}$.

As medidas de área superficial específica foram baseadas no princípio BrunauerEmmett-Teller (BET) (Miranda, 2005). Na análise granulométrica, foi montado um conjunto de peneiras, de 0,250 a 0,0298 mm, e determinou-se a porcentagem de massa de rejeito retida nestas. Para se determinar a basicidade da superfície (Al-Degs et alii ,2000), foram preparados erlenmeyers de $250 \mathrm{~mL}$ contendo 2,0 g do rejeito e 25,0 mL de $\mathrm{HCl} 0,05 \mathrm{~mol} \mathrm{~L}^{-1}$. Os frascos foram agitados por 2 horas. $\mathrm{O}$ sobrenadante foi titulado com $\mathrm{NaOH} 0,05 \mathrm{~mol} \mathrm{~L}^{-1}$. A acidez da superfície foi determinada da mesma forma, acrescentando-se $25,0 \mathrm{~mL}$ de $\mathrm{NaOH} 0,05 \mathrm{~mol} \mathrm{~L}^{-1}$ e o sobrenadante foi titulado com $\mathrm{HCl} 0,05 \mathrm{~mol} \mathrm{~L}^{-1}$. Para a determinação do $\mathrm{pH}$ do rejeito, foi feita a leitura do $\mathrm{pH}$ da água destilada, que foi aferido para 7,0. Em um erlenmeyer de $250 \mathrm{~mL}$, foi colocado $10,0 \mathrm{~g}$ do rejeito e adicionaram-se $25,0 \mathrm{~mL}$ de água destilada com $\mathrm{pH}$ aferido. $\mathrm{O}$ sistema foi agitado por 1 minuto e deixou-se em repouso por 60 minutos. Fez-se a leitura do $\mathrm{pH}$.

\section{Estrutura do corante amarelo remazol}

$\left(\mathrm{C}_{20} \mathrm{H}_{22} \mathrm{~N}_{4} \mathrm{O}_{11} \mathrm{~S}_{3} .2 \mathrm{Na}\right)-$

4-[4,5dihidro-4-[[2-metoxi-5metil-4-[[2-(sulfooxi)etil]sulfonil] fenil]azo-3-metil5-oxo-1H-pirazol-1-il]benzenosulfonato de sódio

\section{Teste para estabelecer 0 tempo de equilíbrio e a massa de adsorvente}

Preparou-se uma solução aquosa de corante puro, na concentração 50,0 $\mathrm{mg} \mathrm{L}^{-1}$. Foram retiradas alíquotas de $50 \mathrm{~mL}$ e transferidas para uma série de erlenmeyers de $250 \mathrm{~mL}$. Aos erlenmeyers foram acrescentados 0,25 , 0,50 e 1,00 grama do adsorvente e foram agitados em diferentes tempos, variando de 0,5 a 6 horas de agitação, até que fosse estabelecido o tempo de equilíbrio do corante. Após a agitação, as soluções foram centrifugadas por 5 minutos a $6000 \mathrm{rpm}$. A seguir, uma alíquota foi retirada e efetuada a leitura de absorbância, em um espectrofotômetro FEMTO 700, no comprimento de onda de máxima absorção do corante (417 nm).

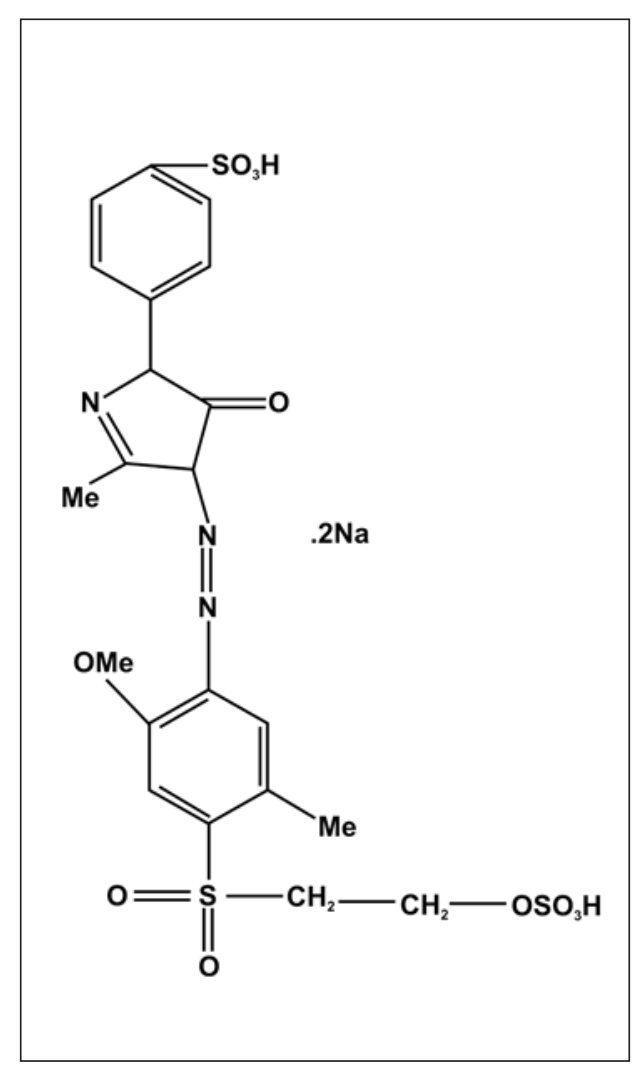

Figura 1 - Estrutura química do corante amarelo remazol (Macedo et alii, 2006). 
Elizabeth Gonçalves Rutz et al.

\section{Teste de adsorção com o corante puro}

Foram preparadas soluções do corante em diversas concentrações a partir da diluição de uma solução-padrão mais concentrada. $\mathrm{O}$ pH das soluções foi ajustado para 4, 7 e 10 pela adição de $\mathrm{HCl}$ ou $\mathrm{NaOH}$ 0,1 mol L-1. Prosseguiram-se os ensaios conforme descrito anteriormente, com a massa de adsorvente e o tempo de agitação estabelecidos.

\section{Isotermas de Adsorção}

Através das leituras de absorbâncias das soluções, obtidas no teste de adsorção com o corante puro, realizouse o ajuste dos dados experimentais conforme o modelo de isoterma de Langmuir, aplicada à adsorção em superfícies completamente homogêneas. Os reagentes que estejam adsorvidos podem, ou não, competir entre si pelos sítios ativos do adsorvente. A isoterma de Langmuir pode ser descrita como a equação linear:

$$
\frac{C e q}{Q e}=\frac{1}{Q o b}+\frac{C e q}{Q o}
$$

Onde:

Ceq: concentração do adsorvato no equilíbrio (mg L-1 ${ }^{-1}$.

Qe: quantidade de material adsorvido por unidade de massa de adsorvente ( $\mathrm{mg} \mathrm{g}^{-1}$ )

Qo: constante de Langmuir relativa à capacidade de adsorção ( $\mathrm{mg} \mathrm{g}^{-1}$ ).

b: constante de Langmuir relativa à constante de equilíbrio ou energia de adsorção ( $\left.\mathrm{L} \mathrm{mg}^{-1}\right)$.

\section{Resultados e discussão}

O pó retido no filtro eletrostático, na fabricação da alumina, tem, como principais constituintes da alumina, pequenas quantidades de carvão $(3,18 \%$ em massa) e traços de óxido de ferro (0,039\%) e de sílica (0,073\%). A Tabela 1 mostra o resultado da análise granulométrica.
A análise granulométrica desse resíduo revela que este é um material muito fino, com a granulometria entre 0,044 e $0,0298 \mathrm{~mm}$. A Tabela 2 mostra os resultados da análise BET do rejeito.

De acordo com a Tabela 2, observa-se um volume total de poros da mesma ordem de grandeza de um carvão ativado com granulometria entre 4,0 e 0,707 mm. Em testes preliminares de adsorção de corantes têxteis realizados com carvão ativado e com o rejeito, observa-se que o pó retido no filtro eletrostático é melhor adsorvente em comparação ao carvão ativado, quando se trata de testes com corantes puros.
A acidez e a basicidade da superfície bem como o pH do rejeito da fabricação da alumina foram determinados. Os resultados mostram uma basicidade e uma acidez, para a superfície, respectivamente iguais a 0,48 e $0,46 \mathrm{mmol}$, por grama de adsorvente, e um $\mathrm{pH}$ de 8,24. Assim, observa-se um maior número de sítios básicos, maior valor da basicidade da superfície e o pH maior do que 7,0.

De acordo com as Tabelas 3, 4 e 5, observa-se que o equilíbrio é atingido em 1 hora de agitação. Com os testes de adsorção realizados, tem-se como resultado uma porcentagem de remoção do corante em torno de $95 \%$, quando é usada uma massa de 0,50 grama do rejeito da fabricação de alumina.

Tabela 1 - Análise granulométrica do rejeito da fabricação da alumina - pó retido no filtro eletrostático.

\begin{tabular}{c|c|c}
\hline Granulometria (malha) & Granulometria (mm) & \% em massa retida \\
\hline 60 & 0,250 & 0 \\
\hline 100 & 0,149 & 0,228 \\
\hline 200 & 0,074 & 2,780 \\
\hline 250 & 0,063 & 2,001 \\
\hline 325 & 0,044 & 64,893 \\
\hline 400 & 0,037 & 17,137 \\
\hline 500 & 0,029 & 11,825 \\
\hline Fundo & $<0,029$ & 1,135 \\
\hline
\end{tabular}

Tabela 2 - Resultados da Análise BET para o rejeito da fabricação da alumina - pó retido no filtro eletrostático.

\section{Adsorção de Nitrogênio Líquido (Técnica BET)}

\begin{tabular}{c|c}
\hline Massa específica $\left(\mathrm{g} / \mathrm{cm}^{3}\right)$ & 2,946 \\
\hline Superfície Específica $\left(\mathrm{m}^{2} / \mathrm{g}\right)$ & 23,46 \\
\hline Tamanho Médio dos Microporos $(\mathrm{nm})$ & 4,985 \\
\hline Volume Total dos Poros $\left(\mathrm{cm}^{3} / \mathrm{g}\right)$ & $5,021 \times 10^{-2}$ \\
\hline Tamanho Máximo dos Poros $(\AA)$ & 1469,6 \\
\hline Diâmetro Médio $(\AA)$ & 85,61 \\
\hline Volume dos Microporos $\left(\mathrm{cm}^{3} / \mathrm{g}\right)$ & $9,817 \times 10^{-3}$ \\
\hline Área dos Microporos $\left(\mathrm{m}^{2} / \mathrm{g}\right)$ & 27,79 \\
\hline
\end{tabular}


Adsorção do corante têxtil amarelo remazol usando um dos rejeitos da fabricação da alumina...

A Figura 2 mostra as isotermas de Langmuir obtidas no processo de linearização do corante amarelo remazol em função do pH.

Observa-se, através da Figura 2, que o modelo de Langmuir se ajusta aos dados experimentais em diferentes valores de $\mathrm{pH}$. Isso pode ser comprovado pelo coeficiente de correlação linear (R), superior a 0,99 nos três valores de $\mathrm{pH}$ estudados. A Tabela 6 resume os resultados obtidos a partir da linearização dos resultados experimentais segundo esse modelo.

Comparando os valores do parâmetro b obtidos para o corante amarelo remazol, observa-se uma maior intensidade de adsorção em $\mathrm{pH}=7$. Segundo o trabalho de Ozacar e Sengil (2002), o pH do ponto de carga zero é registrado como 9,0 para a alumina. Assim tem-se:

$$
\begin{aligned}
& \mathrm{AlOH}_{2}^{+} \stackrel{-\mathrm{H}^{+}}{\longrightarrow} \mathrm{AlOH} \stackrel{-\mathrm{H}^{+}}{\longrightarrow} \mathrm{AlO}^{-} \\
& \mathrm{pH}<9 \ldots \ldots \ldots \ldots . . \mathrm{pH}=9 \ldots \ldots \ldots \ldots \mathrm{pH}>9
\end{aligned}
$$

Em $\mathrm{pH}=4$ ocorrem interações eletrostáticas entre os grupos aniônicos do corante $\left(\mathrm{SO}_{3}^{-}\right)$e os sítios adsorventes do rejeito da fabricação da alumina, carregados positivamente $\left(\mathrm{AlOH}_{2}^{+}\right)$. Entre $\mathrm{pH}=5$ e $\mathrm{pH}=8$, tem-se a interação $\mathrm{AlOH}$ e o corante, através de ligação de hidrogênio. Acima de $\mathrm{pH}=8$, haveria uma menor interação, em virtude da repulsão entre $\mathrm{AlO}^{-}$e o ligante carregado negativamente. De acordo com Dincer, Gune e Karakaya (2007), a baixa adsorção pode ser justificada também por moléculas de corantes que se agregam, dificultando sua difusão para o interior do adsorvente. Em função desses fatores, como encontrado no estudo de adsorção de fluoreto por alumina, o máximo da adsorção ocorreu em pH próximo de 7,0 (Young \& Chiou, 2002).

\section{Conclusão}

De acordo com os objetivos estabelecidos para esse trabalho, observase a aplicabilidade do rejeito da fabricação da alumina, pó retido no filtro eletrostático, na adsorção do corante amarelo remazol. Os resultados mostram,
Tabela 3 - Ensaios de adsorção usando $0,25 \mathrm{~g}$ de rejeito em diferentes tempos de agitação.

\begin{tabular}{c|c|c|c}
\hline Amostra & Tempo (h) & $\mathbf{C}\left(\mathbf{m g ~ L}^{-1}\right)$ & \% Remoção \\
\hline Branco & $\star \star \star$ & 50,83 & $\star \star \star$ \\
\hline 1 & $1 / 2$ & 5,85 & 88,53 \\
\hline 2 & $1 / 2$ & 6,20 & 87,80 \\
\hline 3 & $1 / 2$ & 5,85 & 88,49 \\
\hline 1 & 1 & 5,06 & 90,05 \\
\hline 2 & 1 & 4,26 & 91,62 \\
\hline 3 & 1 & 4,62 & 90,91 \\
\hline 1 & 2 & 4,53 & 91,09 \\
\hline 2 & 2 & 4,79 & 90,58 \\
\hline 3 & 2 & 4,26 & 91,62 \\
\hline 1 & 3 & 3,47 & 93,17 \\
\hline 2 & 3 & 3,21 & 93,68 \\
\hline 3 & 3 & 3,03 & 94,04 \\
\hline
\end{tabular}

Tabela 4 - Ensaios de adsorção usando $0,50 \mathrm{~g}$ de rejeito em diferentes tempos de agitação.

\begin{tabular}{c|c|c|c}
\hline Amostra & Tempo (h) & $\mathbf{C}\left(\mathbf{m g ~ L}^{-1}\right)$ & \% Remoção \\
\hline Branco & $* \star \star$ & 50,83 & $* \star \star$ \\
\hline 1 & $1 / 2$ & 4,09 & 91,95 \\
\hline 2 & $1 / 2$ & 4,18 & 91,78 \\
\hline 3 & $1 / 2$ & 4,44 & 91,27 \\
\hline 1 & 1 & 2,41 & 95,26 \\
\hline 2 & 1 & 2,41 & 95,26 \\
\hline 3 & 1 & 2,68 & 94,73 \\
\hline 1 & 2 & 2,33 & 95,42 \\
\hline 2 & 2 & 2,68 & 94,73 \\
\hline 3 & 2 & 2,59 & 94,9 \\
\hline 1 & 3 & 2,59 & 94,9 \\
\hline 2 & 3 & 2,15 & 95,77 \\
\hline 3 & 3 & 2,68 & 94,73 \\
\hline
\end{tabular}




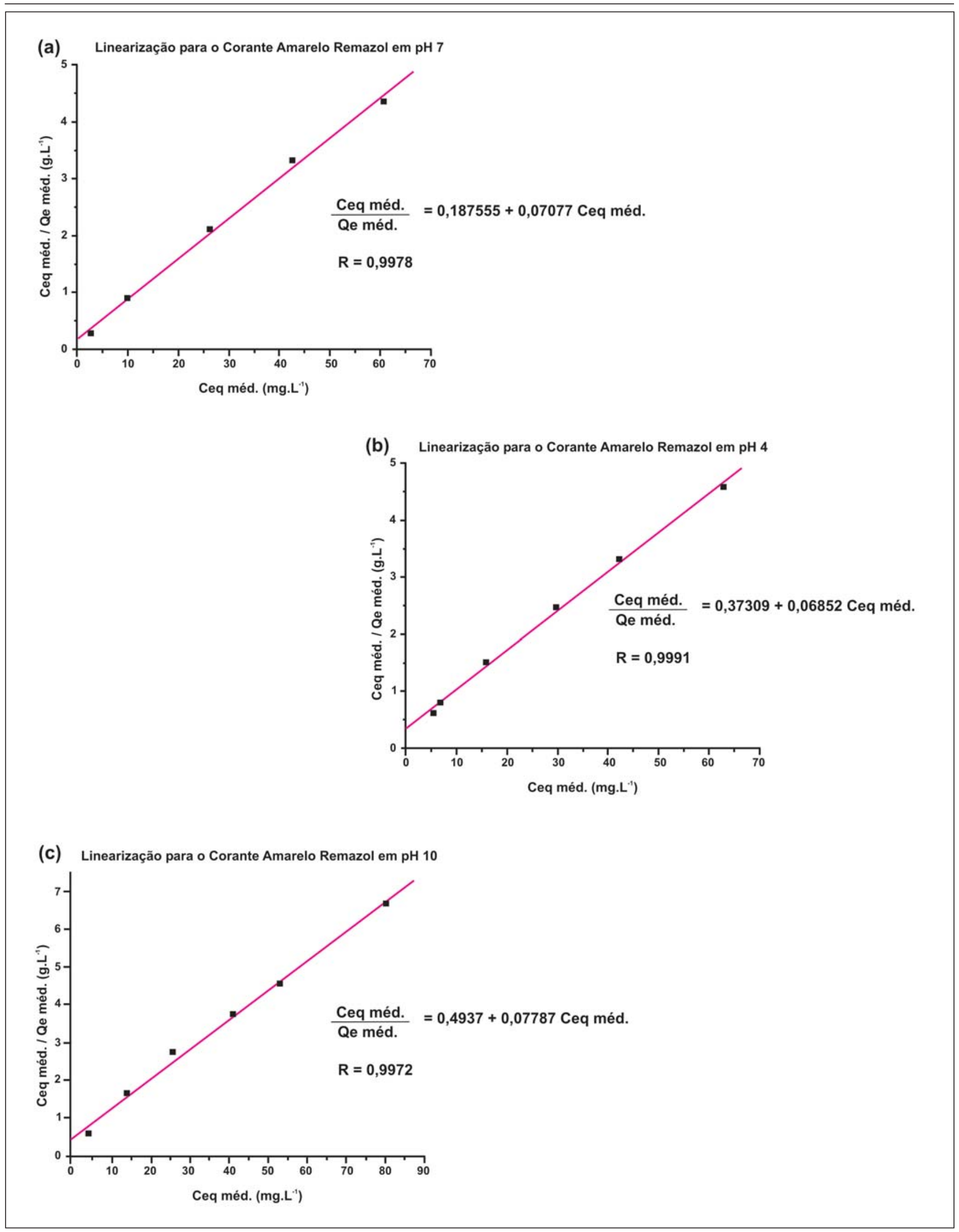

Figura 2 - Linearização do modelo de Langmuir obtido para o corante amarelo remazol em (a) $\mathrm{pH}=7$, (b) $\mathrm{pH}=4 \mathrm{e}(\mathrm{c}) \mathrm{pH}=10$. 
Adsorção do corante têxtil amarelo remazol usando um dos rejeitos da fabricação da alumina...

para uma solução aquosa $50 \mathrm{mg} \mathrm{L}^{-1}$, cerca de 95\% de remoção do corante em solução. O modelo de isoterma de Langmuir mostrou-se adequado para descrever o mecanismo de adsorção desse corante. A capacidade de adsorção do corante depende dos grupamentos químicos existentes na sua estrutura e das interações corante-adsorvente.

Estudos prosseguem no laboratório com outros corantes e também com testes de desorção. Os ensaios de desorção, com solventes orgânicos, visam a recuperar o adsorvente e os corantes (destilação do solvente).

\section{Referências bibliográficas}

AL-DEGS, Y., KHRAISHEH, M. A. M., ALLEN S. J., AHMAD M. N. Effect of carbon surface chemistry on the removal of reactive dyes from textile effluent. Water Resource, v.34, n.3, p.927- 935, 2000.

ALLEN, S. J., McKAY, G., PORTER, J. F. Adsorption isotherm models for basic dye adsorption by peat in single and binary component systems. Journal of Colloid and Interface Science, v.280 p.322-333, 2004.

ANDRADE, F. L. I. Possibilidades de redução da carga poluidora para a indústria de acabamentos de malhas do Estado de Minas Gerais. Belo Horizonte: Programa de Pós-Graduação em Meio Ambiente, Saneamento e Recursos Hídricos/UFMG, 1999. 85f. (Dissertação de Mestrado)

DINCER, A. R., GUNES, Y., KARAKAYA, N. Coal-based bottom ash (CBBA) waste material as adsorbent for removal of textile dyestuffs from aqueous solution. Journal of Hazardous Materials, v.141, p.529-535, 2007.

GONÇALVES, J. F. Rejeito industrial da fabricação da alumina: estudos de adsorção e reciclagem. Belo Horizonte: Programa de Pós-Graduação em Química/ UFMG, 2003. 102f. (Dissertação de Mestrado).

JAIN, A. K. et alii. Utilization of industrial waste products as adsorbents for the removal of dyes. Journal of Hazasdous Materials B, v.101, p.31-38, 2003.

KUNZ, A., MORAES, S., DURÁN, N. Novas tendências no tratamento de efluentes têxteis. Química Nova, v.25, n.1, p.78-820, 2002.

Tabela 5 - Ensaios de adsorção usando $1,00 \mathrm{~g}$ de rejeito em diferentes tempos de agitação.

\begin{tabular}{c|c|c|c}
\hline Amostra & Tempo (h) & $\mathbf{C}\left(\mathbf{m g ~ L}^{-1}\right)$ & \% Remoção \\
\hline Branco & $\star \star \star$ & 50,83 & $\star \star \star$ \\
\hline 1 & $1 / 2$ & 4,09 & 91,95 \\
\hline 2 & $1 / 2$ & 3,91 & 92,31 \\
\hline 3 & $1 / 2$ & 4,44 & 91,27 \\
\hline 1 & 1 & 3,03 & 94,04 \\
\hline 2 & 1 & 3,12 & 93,86 \\
\hline 3 & 1 & 3,03 & 94,04 \\
\hline 1 & 2 & 2,94 & 94,22 \\
\hline 2 & 2 & 3,56 & 93,00 \\
\hline 3 & 2 & 2,94 & 94,22 \\
\hline 1 & 3 & 3,65 & 92,82 \\
\hline 2 & 3 & 3,03 & 94,04 \\
\hline 3 & 3 & 3,03 & 94,04 \\
\hline
\end{tabular}

Tabela 6 - Constantes de Langmuir obtidas pelo processo de linearização do corante amarelo remazol em função do $\mathrm{pH}$ do meio.

\begin{tabular}{c|c|c|c}
\hline \multirow{2}{*}{$\mathbf{p H}$} & \multicolumn{3}{|c}{ Constantes de Langmuir } \\
\cline { 2 - 4 } & Qo $\left(\mathbf{m g ~ g}^{-1}\right)$ & $\mathbf{b}\left(\mathbf{L ~} \mathbf{~ m ~}^{-1}\right)$ & $\mathbf{R}$ \\
\hline 4 & 14,5943 & 0,1837 & 0,9991 \\
\hline 7 & 14,1303 & 0,3773 & 0,9978 \\
\hline 10 & 12,8419 & 0,1577 & 0,9972 \\
\hline
\end{tabular}

LEÃO, M. M. D. et al. Controle ambiental na indústria têxtil: acabamento de malhas. Belo Horizonte: Projeto Minas Ambiente, 2002.

MACEDO, J. S. et alii. Kinetic and calorimetric study of the adsorption of dyes on mesoporous activated carbon prepared from coconut coir dust. Journal of Colloid and Interface Science, v.298, n.2, p.515-522, 2006.

MIRANDA, L. A. Estudo e caracterização de xerogéis de sílica funcionalizados por processos físico-químicos. Belo Horizonte: Programa de Pós-Graduação em Química/UFMG, 2005. 130p. (Tese de Doutorado).
OZACAR, M., SENGIL, I. A. Adsorption of acid dyes from aqueous solutions by calcined alunite and granular activated carbon. Adsorption, v.8, p. 301-308, 2002.

SANTOS, T. C. Resíduos da fabricação da alumina: estudos geoquímicos e reciclagem. Ouro Preto: Programa de Pós-Graduação em Evolução Crustal e Recursos Naturais/UFOP, 1997. 120f. (Dissertação de Mestrado).

YOUNG, K., CHIOU, H. The adsorption of fluoride ion fromaqueous solution by activated alumina. Water, air, and soil pollution, v.133, p.349-360,2002.

Artigo recebido em 16/08/2007 e aprovado em 19/05/2008. 